\title{
Iniciação e consumo de substâncias psicoativas entre adolescentes e adultos jovens de Centro de Atenção Psicossocial Antidrogas/CAPS-AD
}

\author{
Initiation and consumption of psychoactive substances \\ among adolescents and young adults \\ in an Anti-Drug Psychosocial Care Center
}

Carolina Carvalho Silva ${ }^{1}$

Maria Conceição Oliveira Costa ${ }^{1}$

Rosely Cabral de Carvalho ${ }^{1}$

Magali Teresópolis Reis Amaral ${ }^{1}$

Nilma Lázara de Almeida Cruz ${ }^{1}$

Mariana Rocha da Silva ${ }^{1}$

${ }^{1}$ Núcleo de Estudos e Pesquisas na Infância e Adolescência, Departamento de Saúde, Universidade Estadual de Feira de Santana. Av. Transnordestina s/n Campus Universitário Módulo VI, Novo Horizonte. 44.031-460 Feira de Santana BA Brasil. oliveiramco69@gmail.com
Abstract The study seeks to characterize the initiation and consumption pattern of psychoactive substances among adolescents and young adults enrolled in an Alcohol and Drug Psychosocial Care Center (CAPS-AD). This study was conducted with records of attendance and the consumption pattern was classified in accordance with WHO: infrequent use (lifetime use, per year or up to five days per month); frequent use (6 to 19 times in the past 30 days); heavy use ( $\geq 20$ times in the last 30 days). In the age group comparison, the test for proportion and association analysis was used and the prevalence and prevalence ratio was calculated with a significance level of $5 \%$ and $95 \%$ confidence interval. Of the total of adolescents and young adults treated between 2003 and 2008 (475), most were male, single, poorly educated, live with relations and have psychic symptoms. Statistical significance was found for age at initiation of use: adolescents compared to young adults started earlier ( $\leq 14$ years): tobacco, marijuana, cocaine, crack and other SPA consumption. Among adolescents, significant results were found for the less frequent consumption of tobacco, more frequent use of alcohol, and heavy consumption of marijuana. These findings may contribute to the preventive and therapeutic CAPS-AD programs.

Key words Initiation and consumption of psychoactive substances, Adolescents, Young adults
Resumo O estudo tem como objetivo caracterizar a iniciação e o padrão de consumo de substâncias psicoativas, entre adolescentes e adultos jovens matriculados em Centro de Atenção Psicossocial Álcool e Drogas - CAPS AD. Foram utilizados os registros dos atendimentos, sendo o padrão de consumo classificado segundo a OMS: uso pouco frequente (uso na vida, no ano ou no mês até cinco dias); uso frequente (de seis até 19 vezes nos últimos 30 dias); uso pesado ( $\geq 20$ vezes nos últimos 30 dias); na comparação das faixas etárias utilizou-se teste de proporções e para análise das associações, calculou-se prevalência/P e razão de prevalência/RP, com significância de 5\% e intervalo de confiança 95\%. No total de adolescentes e jovens atendidos entre 2003 e 2008 (475), a maioria foi masculina, solteira, com baixa escolaridade, coabitando com pais e sintomas psíquicos. Constatou-se significância estatística para idade de iniciação ao consumo: adolescentes, em relação aos jovens iniciaram mais precocemente ( $\leq 14$ anos): o consumo de tabaco; maconha, cocaina; crack outras SPA. Entre adolescentes, verificou-se resultados significantes para consumo menos frequente de tabaco; mais frequente de álcool; e consumo pesado de maconha. Esses achados podem contribuir para programas preventivos e terapêuticos do CAPS-AD.

Palavras-chave Iniciação e consumo de substâncias psicoativas, Adolescente, Adulto jovem 


\section{Introdução}

Segundo estudiosos, o aumento significativo do uso de substâncias psicoativas (SPA) entre adolescentes e jovens e a precocidade dessa prática representam desafios para a saúde pública, em função da forte relação com outros agravos ambientais que comprometem a saúde e a vida, apontando alta vulnerabilidade destes grupos etários ${ }^{1}$.

$\mathrm{Na}$ adolescência, fase da vida marcada por transformações psicossociais e busca da autonomia, os eventos negativos, tais como falta de suporte familiar e social, condições socioeconômicas precárias, desvantagem educacional, entre outros fatores, aumentam a vulnerabilidade ao uso de SPA e outros agravos associados ao consumo dessas substâncias. As ambivalências que gratificam e incomodam, podem gerar conflitos geracionais e psicossociais com a família e o ambiente ${ }^{2,3}$.

De acordo com a Organização Mundial de Saúde (OMS), cerca de $10 \%$ das populações dos centros urbanos, em nível mundial, consomem abusivamente SPA, independente da idade, sexo, nível de instrução e poder aquisitivo, sendo o álcool a substância mais consumida ${ }^{4}$. No Brasil, no que diz respeito ao consumo dessas substâncias entre adolescentes e jovens, o padrão revelase qualitativamente distinto, a partir de referências culturais e características particulares de comportamentos ${ }^{5}$.

Os três levantamentos realizados pelo Centro Brasileiro de Informações sobre Drogas Psicotrópicas (CEBRID) com populações estudantis de dez capitais brasileiras, nos períodos de 1987, 1989, 1993 e 1997 constituem um amplo banco de dados sobre a problemática do uso de SPA, revelando aumento significativo do consumo precoce de algumas substâncias. Entretanto, cabe enfatizar que a generalização dos resultados desses estudos merece cautela, visto que a prevalência desta prática pode variar, conforme peculiaridades regionais, o que implica a necessidade de planejamentos e intervenções a partir de pesquisas específicas, no intuito de adotar estratégias adequadas à realidade de cada grupo populacional e ambiente cultural ${ }^{5}$. Estes estudos também não refletem a realidade dos adolescentes e jovens fora da escola.

O reconhecimento das particularidades da adolescência e juventude impõe desafios à elaboração e execução de políticas de saúde e estratégias terapêuticas específicas e amplas, quanto à prevenção e intervenção do uso de SPA. Nesta pers- pectiva, a nível nacional, tem sido implementada a proposta de atenção aos usuários de SPA, através dos Centros de Atenção Psicossocial, especializados no atendimento dos usuários que sofrem consequências do uso/abuso de SPA (CAPS $\mathrm{AD})$. Estes serviços integram e fortalecem o processo da Reforma Psiquiátrica e a construção de políticas de atenção integral à saúde mental.

Os CAPS AD organizam a atenção, a partir de doze anos de idade, oferecendo atendimento individualizado, de evolução contínua, através de atividades individuais e grupais, com estratégias terapêuticas que visam à reabilitação psicossocial dos usuários, fortalecimento dos vínculos (familiares e sociais) e incentivo ao processo de autonomia do sujeito ${ }^{4}$. A convivência dos profissionais de saúde e as dificuldades enfrentadas no tratamento, adesão e reabilitação de usuários de SPA, principalmente entre adolescentes e adultos jovens do CAPS AD têm servido de estímulo para a busca de novos conhecimentos e aprofundamento da temática, principalmente quanto aos fatores que contribuem para o uso precoce e abusivo nesta fase de vida, considerando as repercussões individuais, contexto familiar e social.

A relevância do estudo está diretamente relacionada à importância do CAPS $\mathrm{AD}$, como estratégia de atendimento em saúde mental utilizando recursos terapêuticos grupais e individuais, para os usuários, familiares e comunidade, assim como estar sediado em um município de referência, para a população da região do semiárido e sertão do Estado da Bahia. O CAPS AD de Feira de Santana representa uma referência para o atendimento clínico e psicossocial de usuários de SPA, no município e região semiárida. Feira de Santana é a segunda maior cidade do Estado (cerca de 600 mil habitantes), localizada às margens de um importante entroncamento rodoviário (rodovias federais e estaduais), que faz ligação entre as regiões norte, nordeste e sudeste do país, destacando-se pela presença de um importante polo industrial do Estado e como portal do semiárido da Bahia, com intenso fluxo migratório das populações pobres do sertão baiano.

O objetivo deste estudo foi caracterizar a iniciação e o padrão de consumo de SPA, entre adolescentes e adultos jovens matriculados no CAPS AD de Feira de Santana, Bahia.

\section{Método}

Este é um estudo de caráter retrospectivo, realizado com população da faixa compreendida en- 
tre 12 - 24 anos (adolescentes e adultos jovens) atendidos no CAPS AD de Feira de Santana, no período 2003 a 2008. Foram utilizados dados dos prontuários, para investigação dos aspectos sociodemográficos, características da procura para tratamentos, padrões de iniciação e consumo de SPA lícitas e ilícitas nesse período.

As variáveis foram agrupadas, segundo objetivos do estudo: 1) dados sociodemográficos: sexo, faixa etária adolescente (12-19 anos) e adultos jovens (20-24 anos); estado civil; coabitação (família e outros); nível de escolaridade e prática religiosa; 2) motivos da procura ao CAPS AD, queixas e tratamentos; 3 ) idade da iniciação ao consumo das SPA; 4) padrão de consumo das diferentes SPA. Para análise da idade de iniciação de SPA foram consideradas duas faixas etárias $\leq$ 14 anos e 15-19 anos. Para caracterizar o padrão de consumo foi utilizada a classificação da OMS/ WHO: uso na vida; uso no ano; uso no mês (nos últimos 30 dias); uso frequente ( $\geq 6$ vezes nos últimos 30 dias); uso pesado ( $\geq 20$ vezes nos últimos 30 dias); uso abusivo (padrão que tenha causado dano à saúde física ou mental sem que a pessoa preencha os critérios estabelecidos para dependência) ${ }^{6}$. Com base nessa classificação, foram utilizadas apenas três categorias de uso: uso pouco frequente (uso na vida, no ano ou no mês até cinco dias); uso frequente (de seis até 19 vezes nos últimos 30 dias); uso pesado ( $\geq 20$ vezes nos últimos 30 dias).

Os dados foram processados eletronicamente com a utilização do Programa StatisticalPackage for Social Science (SPSS), versão 10.0 for Windows (1998). Foram calculadas frequências absolutas e proporcionais das variáveis sociodemográficas, motivos de procura ao serviço e tratamento realizado. As idades de iniciação $(\leq 14$ anos e 15-19 anos) e os padrões de consumo (menos frequente, frequente; pesado/abusivo) foram analisados por faixas etárias (12-19 e 2024 anos), segundo os diferentes tipos de drogas lícitas e ilícitas. Para comparar as faixas etárias (12-19 e 20-24), utilizou-se o teste de proporções ( $p$ valor $<0,05$ ); para analisar associações, calculou-se prevalência/P e razão de prevalência/RP, com nível de significância de 5\% e intervalos de confiança 95\%. Esse estudo foi aprovado no Comitê de Ética e Pesquisa da Universidade Estadual de Feira de Santana.

\section{Resultados}

No período estudado, foram atendidos 475 adolescentes e adultos jovens, sendo que a maioria era do sexo masculino e coabitava com os pais Adolescentes destacam-se por mais alta prevalência de baixa escolaridade ( $\leq 8$ séries), ser solteiro e não ter religião $(\mathrm{p}<0,001)$ (Tabela 1$)$.

Quanto ao motivo da procura de atendimento (Tabela 2), as faixas etárias apresentaram diferenças significantes, para o tipo de encaminhamento ao CAPS AD, onde os adultos jovens mostraram maiores proporções da demanda espontânea e os adolescentes, encaminhados por Instituições do Sistema Judiciário $(\mathrm{p}<0,001)$. Cerca de $50 \%$ de ambas as faixas etárias apresentavam sintomas psíquicos dificuldades emocionais relacionadas ao uso como, por exemplo, alucinações, problemas de relacionamento interpessoal, insônia e medos; em torno de 1/3 dos adolescentes e jovens relataram sintomas clínicos como náuseas, dores, pressão arterial alta. A maioria absoluta não tinha realizado tratamento especializado relacionado ao uso de substâncias, mas tinha frequentado grupo de ajuda em comunidade terapêutica. A demanda para tratamento mais constatada nos dois grupos é a abstinência ao uso de substâncias.

No que se refere à idade de iniciação do consumo de SPA (Tabela 3), as análises da Razão de Prevalência (RP) apontaram associações significantes na comparação entre as faixas etárias. Dentre aqueles que iniciaram o consumo de SPA até 14 anos de idade constata-se que os adolescentes, comparados ao grupo de jovens, predominam no consumo de tabaco (RP 1,47 e IC 1,17$1,86)$ e de substâncias ilícitas, como maconha (RP 1,90 e IC 1,48-2,43), cocaína (RP 2,39 e IC 1,332,31); crack (RP 3,89 e IC 1,97-7-67) e outras SPA como, por exemplo, anabolizantes e medicamentos (RP2,00 e IC 1,03-3,86). Dentre aqueles que iniciaram o consumo de SPA após os 14 anos, constatou-se a iniciação mais precoce de crack entre adolescentes na faixa dos 15-19 anos (RP 1,26 e IC 1,00 e 1,59), se comparados aos jovens.

Com relação ao padrão de consumo de SPA (Tabela 4), as análises mostraram resultados significantes entre as faixas etárias, para o consumo de tabaco, álcool e maconha. Comparados aos adultos jovens (20-24 anos), os adolescentes (12 a 19 anos) predominam entre aqueles com uso menos frequente de tabaco (RP7,14 e IC 4,13$12,32)$, consumo frequente de álcool (RP1,42 e IC 1,04-1,96) e consumo pesado/abusivo de maconha (RP1,16 e IC 1,02-1,31). Jovens destacamse dentre os que fazem uso pesado de álcool (RP 0,62 e IC 0,41-0,96) e uso pouco frequente de maconha (RP 0,43 e IC 0,20-0,95). 
Tabela 1. Características sócio-demográficas de adolescentes e adultos jovens matriculados no CAPS AD, Feira de Santana, Bahia, 2003-2008.

\begin{tabular}{|c|c|c|c|c|c|}
\hline \multirow{3}{*}{ Variáveis sócio-demográficas } & \multicolumn{4}{|c|}{ Faixa etária (anos) } & \multirow{3}{*}{ P valor ${ }^{* *}(<0,05)$} \\
\hline & \multicolumn{2}{|c|}{12 a 19} & \multicolumn{2}{|c|}{20 a 24} & \\
\hline & $\mathbf{N}$ & $\%$ & $\mathbf{N}$ & $\%$ & \\
\hline \multicolumn{6}{|l|}{ Sexo } \\
\hline Masculino & 193 & 85,8 & 222 & 88,8 & 0,441 \\
\hline Feminino & 32 & 14,2 & 28 & 11,2 & 0,968 \\
\hline Total & 225 & 100,0 & 250 & 100,0 & \\
\hline \multicolumn{6}{|l|}{ Estado Civil } \\
\hline Solteiro & 214 & 95,1 & 204 & 81,9 & $<0,001^{*}$ \\
\hline Outros $^{\mathrm{a}}$ & 11 & 4,9 & 45 & 18,1 & 0,535 \\
\hline Total & 225 & 100,0 & 249 & 100,0 & \\
\hline \multicolumn{6}{|l|}{ Coabitação atual } \\
\hline Pai e Mãe & 239 & 53,8 & 239 & 60,4 & 0,172 \\
\hline Outros $^{\mathrm{b}}$ & 205 & 46,2 & 157 & 39,6 & 0,251 \\
\hline Total & 444 & 100,0 & 396 & 100,0 & \\
\hline \multicolumn{6}{|l|}{ Escolaridade } \\
\hline$\leq 8^{\mathrm{a}}$ série $^{\mathrm{c}}$ & 192 & 85,3 & 143 & 57,7 & $<0,001^{*}$ \\
\hline$\geq 2^{\circ} \mathrm{grau}^{\mathrm{d}}$ & 32 & 14,2 & 105 & 42,3 & $0,007^{*}$ \\
\hline Total & 225 & 100,0 & 248 & 100,0 & \\
\hline \multicolumn{6}{|l|}{ Prática Religiosa } \\
\hline Nenhuma & 130 & 60,2 & 83 & 34,6 & $<0,001^{*}$ \\
\hline Alguma $^{e}$ & 86 & 39,8 & 157 & 65,4 & $<0,001^{*}$ \\
\hline Total & 216 & 100,0 & 240 & 100,0 & \\
\hline
\end{tabular}

*Estatisticamente significante; ** teste de proporção entre faixas etárias (12-19 e 20-24)

a Separado(a)/Divorciado(a) $(\mathrm{n}=3)$; Viúvo(a) $(\mathrm{n}=1)$; Casado/ União Livre $(\mathrm{n}=52)$

${ }^{b}$ Habita sozinho $(n=11)$, irmãos $(n=121)$, parentes $(n=76)$ e filhos $(n=52)$; Amigos, pensão/república, centro de recuperação, Melo Matos; Companheiro $(n=53)$; ${ }^{c}$ Analfabetos $(n=4)$; Ensino Fundamental - $1^{\text {a }}$ a $4^{\text {a }}$ série $\left.=91\right)$; Ensino Fundamental - $5^{\mathrm{a}}$ a $8^{\mathrm{a}}$ série $(\mathrm{n}$ $=240) ;{ }^{\mathrm{d}}$ Ensino médio $(\mathrm{n}=124)$; Superior $(\mathrm{n}=13) ;{ }^{\mathrm{e}}$ Espírita $(\mathrm{n}=3)$; Protestante/ Evangélica $(\mathrm{n}=95) ;$ Católica $(\mathrm{n}=138)$.

\section{Discussão}

O uso de substâncias psicoativas na adolescência e juventude tem sido uma preocupação coletiva da contemporaneidade pela relevância social do tema, assim como pelo aumento significativo dos índices de consumo, reforçando a gravidade do problema. Essa temática tem mobilizado esforços, no sentido da produção de conhecimento sobre o fenômeno, assim como na busca de soluções que possam contribuir para minimizar danos, especialmente nos grupos mais vulneráveis, como a adolescência e juventude ${ }^{7}$.

Em nível mundial, estudos têm revelado alta prevalência de uso de SPA em faixa etária cada vez mais precoce, apontando a importância das intervenções nesse grupo ${ }^{8}$.

Os achados do presente estudo, especificamente voltados para o público adolescente e jovem, constatam os altos índices da iniciação muito precoce ao consumo de drogas lícitas e ilícitas, nas faixas da adolescência, reforçando os dados apresentados na literatura. Destacam a crescente necessidade de intervenções nas faixas mais jovens, pelas características do desenvolvimento psicossocial e vulnerabilidades peculiares deste grupo ${ }^{9}$. Estudos realizados pelo Instituto LatinoAmericano das Nações Unidas para a Prevenção do Delito e Tratamento (ILANUD) têm mostrado associação entre uso de SPA e infrações penais cometidas por adolescentes. No Brasil, onde a maioria (acima de 70\%) das infrações é contra a propriedade, faz-se importante ressaltar a necessidade de redução no uso de SPAS, através da participação dos setores envolvidos, na prevenção e encaminhamento, como a escola; assim como dos centros especializados, em particular o CAPS-AD, que representam importantes instâncias de atendimento e acompanhamento dos casos que requerem atuação psicossocial e de saúde, ampliando o atendimento ao debate das questões sociais, políticas e educacionais, impor- 
Tabela 2. Motivos da procura, queixas e demandas para o tratamento de adolescentes e adultos jovens matriculados no CAPS AD, Feira de Santana, Bahia, 2003-2008.

\begin{tabular}{|c|c|c|c|c|c|}
\hline & \multicolumn{4}{|c|}{ Faixa etária (anos) } & \multirow{3}{*}{$P$ valor ${ }^{* *}(<0,05)$} \\
\hline & \multicolumn{2}{|c|}{12 a 19} & \multicolumn{2}{|c|}{20 a 24} & \\
\hline & $\mathbf{N}$ & $\%$ & $\mathbf{N}$ & $\%$ & \\
\hline \multicolumn{6}{|l|}{ Motivo para procura } \\
\hline Demanda espontânea $^{a}$ & 122 & 50,6 & 189 & 73,0 & $<0,001^{\star}$ \\
\hline Encaminhado por Instituições Judiciais & 112 & 46,5 & 68 & 26,2 & $0,011^{*}$ \\
\hline Outros & 7 & 2,9 & 2 & 0,8 & $0,015^{*}$ \\
\hline Total & 241 & 100,0 & 259 & 100,0 & \\
\hline \multicolumn{6}{|l|}{ Queixa no momento do acolhimento } \\
\hline Sintomas psíquicos ${ }^{\mathrm{b}}$ & 133 & 49,8 & 154 & 49,4 & 0,959 \\
\hline Sintomas clínicos ${ }^{c}$ & 79 & 29,6 & 108 & 34,6 & 0,573 \\
\hline Sem queixas ${ }^{\mathrm{d}}$ & 55 & 20,6 & 50 & 16,0 & 0,722 \\
\hline Total & 267 & 100,0 & 312 & 100,0 & \\
\hline \multicolumn{6}{|l|}{ Tratamento anterior ao uso de drogas } \\
\hline Sim & 41 & 19,3 & 67 & 27,7 & 0,451 \\
\hline Não & 171 & 80,7 & 175 & 72,3 & 0,086 \\
\hline Total & 212 & 100,0 & 242 & 100,0 & \\
\hline \multicolumn{6}{|l|}{ Tipo de tratamento } \\
\hline Psiquiátrico/Psicoterápico ${ }^{\mathrm{e}}$ & 23 & 45,1 & 22 & 29,7 & 0,448 \\
\hline Comunidade Terapêutica/ Grupo de ajuda ${ }^{\mathrm{f}}$ & 28 & 54,9 & 52 & 70,3 & 0,258 \\
\hline Total & 51 & 100,0 & 74 & 100,0 & \\
\hline \multicolumn{6}{|l|}{ Demandas para o tratamento } \\
\hline Abstinência ${ }^{g}$ & 166 & 84,3 & 198 & 81,5 & 0,572 \\
\hline Outras $^{\mathrm{h}}$ & 31 & 15,7 & 45 & 18,5 & 0,993 \\
\hline Total & 197 & 100,0 & 243 & 100,0 & \\
\hline
\end{tabular}

"Estatisticamente significante; ${ }^{* *}$ teste de proporção entre faixas etárias (12-19 e 20-24); ${ }^{a}$ Família, parentes e amigos ( $\left.\mathrm{n}=166\right)$; ${ }^{b}$ Sintomas psíquicos: dificuldades emocionais relacionadas ao uso, como alucinações, problemas de relacionamento interpessoal, insônia, medos entre outros; ' Sintomas clínicos: episódios orgânicos como náuseas, dores, cirrose, pressão elevada entre outros;

${ }^{\mathrm{d}}$ Sem queixas $(\mathrm{n}=74) ;{ }^{\mathrm{e}}$ Médico clínico $(\mathrm{n}=2)$; Psicoterápico $(\mathrm{n}=10)$; Psicanalítico $(\mathrm{n}=2) ;{ }^{\mathrm{f}}$ Grupo de ajuda mútua $(\mathrm{n}=5)$; Religioso ( $\mathrm{n}=4)$; Outros $(\mathrm{n}=8) ;{ }^{\mathrm{g}}$ Abstinência: abandonar, abster-se ao uso das drogas; ${ }^{\mathrm{h}}$ Redução de danos $(\mathrm{n}=35)$ e Familiar $(\mathrm{n}=7)$.

tantes aspectos ligados à redução das infrações juvenis, do tráfico de drogas e do uso precoce e abusivo de SPA entre jovens.

De acordo com a Organização Mundial da Saúde (2004) e estudos realizados pelo CEBRID ${ }^{10-}$ ${ }^{13} \mathrm{o}$ consumo de SPA, principalmente as substâncias ilícitas, constitui uma prática predominantemente masculina, corroborando os achados encontrados no CAPS AD de Feira de Santana, com $80 \%$ dos adolescentes e adultos jovens atendidos do sexo masculino ${ }^{14,15}$. Para a região do semiárido e o sertão da Bahia, a atuação de um Centro de Atenção psicossocial em município de referência, Feira de Santana, representa uma importante ferramenta de prevenção e enfrentamento da drogadição. Neste contexto, o encaminhamento de adolescentes e jovens a esse serviço de referência por Instituições do Sistema Judiciário representa uma tentativa de desconstrução do sofrimento psíquico e do isolamento dessa população mais vulnerável de nossa sociedade, integrando novas bases clínicas ao acolhimento e cuidados à saúde mental, estratégia fundamental para o tratamento e reintegração social na adolescência e juventude.

O desequilíbrio entre escolaridade e faixa etária detectado nessa pesquisa, onde a maioria do grupo de 12-19 anos cursava o $1^{\circ}$ grau, pode estar sinalizando possível relação de causa ou consequência com o consumo de SPA. O baixo nível de escolarização e a dificuldade de engajamento na escola têm sido apontados como potenciais fatores de vulnerabilidade entre adolescentes e adultos jovens, considerando que contribui de forma incisiva para a perpetuação deste ciclo, interferindo na inserção e permanência na escola e trabalho; conflitos nas relações pessoais e familiares, desencadeando condutas antissoci- 
Tabela 3. Prevalência (P), Razão de Prevalência (RP) e Intervalo de Confiança (IC) da idade de iniciação ao consumo das SPA, segundo faixa etária dos adolescentes e adultos jovens matriculados no CAPS AD. Feira de Santana, Bahia, 2003-2008.

\begin{tabular}{|c|c|c|c|c|c|c|c|}
\hline \multirow{3}{*}{ SPA / Faixa etária(anos) } & \multirow[b]{3}{*}{$\mathbf{N}$} & \multicolumn{6}{|c|}{ Idade de iniciação ao consumo de SPA } \\
\hline & & \multicolumn{3}{|c|}{$\leq 14$ anos $^{\mathrm{a}}$} & \multicolumn{3}{|c|}{15 a 19 anos $^{a}$} \\
\hline & & $\mathbf{P}$ & $\mathbf{R P}$ & IC $95 \%$ & $\mathbf{P}$ & $\mathbf{R P}$ & IC 95\% \\
\hline \multicolumn{8}{|l|}{ Álcool } \\
\hline 15 a 19 & 97 & 52,6 & 1,20 & $0,93-1,56$ & 47,4 & 1,15 & $0,89-1,48$ \\
\hline 20 a 24 & 165 & 43,6 & 1,00 & - & 54,5 & 1,00 & - \\
\hline \multicolumn{8}{|l|}{ Tabaco } \\
\hline 15 a 19 & 135 & 60,0 & 1,47 & $1,17-1,86^{*}$ & 40,0 & 0,71 & $0,54-0,92$ \\
\hline 20 a 24 & 162 & 40,7 & 1,00 & - & 55,5 & 1,00 & \\
\hline \multicolumn{8}{|l|}{ Maconha } \\
\hline 15 a 19 & 164 & 62,2 & 1,90 & $1,48-2,43^{*}$ & 37,8 & 0,66 & $0,53-0,83$ \\
\hline 20 a 24 & 168 & 32,7 & 1,00 & - & 57,7 & 1,00 & \\
\hline \multicolumn{8}{|l|}{ Cocaína } \\
\hline 15 a 19 & 68 & 33,8 & 2,39 & $1,33-4,31^{*}$ & 66,1 & 1,15 & $0,90-1,46$ \\
\hline 20 a 24 & 99 & 14,1 & 1,00 & - & 57,6 & 1,00 & - \\
\hline \multicolumn{8}{|l|}{ Crack } \\
\hline 15 a 19 & 103 & 34,0 & 3,89 & $1,97-7,67^{*}$ & 66,0 & 1,26 & $1,00-1,59^{*}$ \\
\hline 20 a 24 & 103 & 8,7 & 1,00 & - & 52,4 & 1,00 & - \\
\hline \multicolumn{8}{|c|}{ Anabolizantes, solventes e outros } \\
\hline 15 a 19 & 43 & 37,2 & 2,00 & $1,03-3,86^{*}$ & 62,8 & 1,00 & $0,74-1,36$ \\
\hline 20 a 24 & 59 & 18,6 & 1,00 & - & 62,7 & 1,00 & - \\
\hline
\end{tabular}

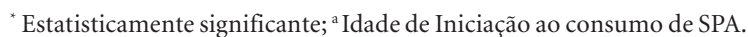

Nota: Foram excluídos da tabela os adolescentes na faixa de 12 a 14 anos, que tiveram o seguinte perfil de uso: álcool (14), tabaco (11), maconha (12), cocaína (7), crack (8) e outros (9).

Tabela 4. Prevalência (P), Razão de Prevalência (RP) e Intervalo de Confiança (IC) do Padrão de Consumo das SPA, segundo tipo de SPA e faixa etária dos adolescentes e adultos jovens matriculados no CAPS AD. Feira de Santana, Bahia, 2003-2008.

\begin{tabular}{|c|c|c|c|c|c|c|c|c|c|c|}
\hline \multirow{3}{*}{ SPA / Faixa etária(anos) } & \multirow[b]{3}{*}{$\mathbf{N}$} & \multicolumn{8}{|c|}{ Padrão de Consumo de SPA } & \\
\hline & & \multicolumn{3}{|c|}{ Menos frequente } & \multicolumn{3}{|c|}{ Uso frequente } & \multicolumn{3}{|c|}{ Uso pesado } \\
\hline & & $\mathbf{P}$ & $\mathbf{R P}$ & IC $95 \%$ & $\mathbf{P}$ & $\mathbf{R P}$ & IC $95 \%$ & $\mathbf{P}$ & $\mathbf{R P}$ & IC $95 \%$ \\
\hline \multicolumn{11}{|l|}{ Álcool } \\
\hline 12 a 19 & 111 & 11,2 & 1,02 & $0,66-1,57$ & 68,4 & 1,42 & $1,04-1,96^{*}$ & 20,4 & 0,62 & $0,41-0,96^{*}$ \\
\hline 20 a 24 & 164 & 12,2 & 1,00 & & 54,9 & 1,00 & - & 32,9 & 1,00 & - \\
\hline \multicolumn{11}{|l|}{ Tabaco } \\
\hline 12 a 19 & 129 & 8,3 & 7,14 & $4,13-12,32^{*}$ & 6,7 & 1,12 & $0,69-1,82$ & 85,0 & 0,93 & $0,86-1,02$ \\
\hline 20 a 24 & 160 & 3,1 & 1,00 & - & 5,6 & 1,00 & & 91,3 & 1,00 & - \\
\hline \multicolumn{11}{|l|}{ Maconha } \\
\hline 12 a 19 & 149 & 3,6 & 0,43 & $0,20-0,95^{*}$ & 12,9 & 0,87 & $0,62-1,24$ & 83,6 & 1,16 & $1,02-1,31^{*}$ \\
\hline 20 a 24 & 137 & 11,7 & 1,00 & - & 16,1 & 1,00 & - & 72,3 & 1,00 & - \\
\hline \multicolumn{11}{|l|}{ Cocaína } \\
\hline 12 a 19 & 65 & 27,1 & 0,86 & $0,57-1,31$ & 45,8 & 0,88 & $0,63-1,22$ & 27,1 & 1,89 & $0,98-3,64$ \\
\hline 20 a 24 & 82 & 32,9 & 1,00 & - & 52,4 & 1,00 & - & 14,6 & 1,00 & - \\
\hline \multicolumn{11}{|l|}{ Crack } \\
\hline 12 a 19 & 97 & 16,1 & 1,12 & $0,80-1,58$ & 26,4 & 1,09 & $0,81-1,47$ & 57,5 & 0,92 & $0,73-1,16$ \\
\hline 20 a 24 & 86 & 13,9 & 1,00 & - & 23,2 & 1,00 & - & 62,8 & 1,00 & - \\
\hline \multicolumn{11}{|c|}{ Anabolizantes, solventes e outros } \\
\hline 12 a 19 & 54 & 59,5 & 0,87 & $0,58-1,29$ & 16,6 & 1,27 & $0,78-2,07$ & 23,8 & 1,10 & $0,57-2,13$ \\
\hline 20 a 24 & 64 & 67,2 & 1,00 & - & 10,9 & 1,00 & - & 21,9 & 1,00 & - \\
\hline
\end{tabular}

"Estatisticamente significante. 
ais, aumento do uso e envolvimento com o narcotráfico. A relação entre prejuízo escolar (atraso ou abandono) e uso de SPA tem sido abordada em vários estudos, sendo citada como uma das importantes consequências ${ }^{16}$.

A pesquisa realizada não permite avaliar relação de causalidade entre consumo de SPA e escolaridade, muito embora a alta prevalência do desequilíbrio entre escolaridade e faixa etária possa indicar esta direção. O IV levantamento sobre o uso de drogas entre estudantes, realizados em capitais brasileiras, apontou associação entre uso de SPA e o baixo rendimento escolar ${ }^{12}$.

No que se refere à procura para atendimento no CAPS AD, o presente estudo mostrou que a proporção do encaminhamento judicial foi superior entre adolescentes, enquanto a demanda espontânea mais frequente entre adultos jovens, resultados estes que corroboram com estudos que abordam o desenvolvimento psicossocial na adolescência e juventude. A literatura mostra que, na adolescência inicial e intermediária (10-16 anos), o indivíduo possui baixa capacidade de percepção dos reais riscos e vulnerabilidades. Nesta fase, adolescentes buscam independência e identificação com o grupo de iguais, justificando a influência de amigos ao consumo de SPA. Com $\mathrm{o}$ avançar da idade e a maturidade aumentam as chances da avaliação quanto aos riscos e necessidade de proteção ${ }^{17}$.

Ainda em relação aos encaminhamentos para o CAPS AD, salienta-se que, o consumo de SPA ilícita, como maconha, crack e cocaína, constituem prática proibida no Brasil e sua utilização pode ser interpretada pela justiça como violação judicial. Os resultados do presente estudo confirmam pesquisas realizadas em outros contextos, com adolescentes usuários, onde é ressaltado que o envolvimento com a justiça precede a abordagem pelo Sistema de Saúde ${ }^{9,16,18,19}$. Os resultados de Feira de Santana convergem com esses achados. É possível que as altas proporções de adolescentes encaminhados para o CAPS AD por via judicial, para cumprimento de medidas educativas, sejam consequência de comportamentos indesejados socialmente relacionados ao consumo de SPA, como, por exemplo, a realização de pequenos delitos que possibilitem a aquisição das drogas.

Entre as motivações para procura do CAPS $\mathrm{AD}$, destaca-se o importante papel da família de adolescentes e adultos jovens, conforme foi verificado no presente estudo. Estes resultados concordam com outras pesquisas que apontam a família como principal responsável por esta de- manda, em busca de apoio psicológico e tratamento clínico. Assim, vale assinalar que, ao mesmo tempo em que a família é responsável pelo cuidado, diante do envolvimento e consumo de drogas, pode representar fator de influência significativa quanto à projeção de vida destes ${ }^{2}$.

É importante ainda ressaltar que, na atenção especializada a adolescentes e jovens usuários de SPA, considerando as demandas características desse grupo, deve ser priorizada a equipe multidisciplinar, com planos terapêuticos de atendimento e intervenção que fortaleçam os vínculos e ampliem o diálogo, através de estratégias inovadoras de inclusão e relato de experiências de ex-usuários.

De acordo com pesquisas, as influências ambientais representam fatores importantes e preponderantes para a iniciação no uso de SPA, destacando a pressão dos amigos, hábitos familiares, os quais contribuem para o contato precoce com SPA, aumentando a probabilidade de continuidade do consumo e riscos futuros. Estes achados reforçam o entendimento de que a família está implicada no desenvolvimento de seus membros. Fatores familiares têm se destacado como possíveis determinantes de risco e proteção de adolescentes e adultos, cabendo assinalar que o funcionamento familiar coeso e adaptado correlaciona-se de forma positiva com fatores de prevenção e proteção para essas práticas. O estabelecimento de regras e limites pela família é destacado como parâmetro fundamental para que jovens assumam responsabilidades pelas escolhas e $\operatorname{atos}^{20}$.

Quanto à idade de iniciação ao consumo, pesquisas apontam que o álcool e o tabaco são as drogas mais precocemente utilizadas, principalmente quando esta prática ocorre na adolescência. Contudo, a maconha é a droga de iniciação eleita entre as ilícitas, com início entre 12 e 16 anos de idade, muito embora possam iniciar com drogas mais potentes ${ }^{18}$. Levantamentos epidemiológicos sobre o consumo de SPA entre jovens, em nível mundial, assim como no Brasil, ressaltam que a iniciação vem ocorrendo na passagem da infância para a adolescência, trazendo riscos adicionais, em decorrência da vulnerabilidade desta fase $\mathrm{e}^{21,22}$.

No município estudado, foi verificado que a faixa etária da adolescência mostrou resultados significantes, quanto à iniciação ao consumo de tabaco e das SPA ilícitas (cocaína, crack e maconha) em idade muito precoce ( $<14$ anos). Esses achados corroboram com outros estudos, com crianças e adolescentes usuários, nos quais foi 
observado o uso precoce de substâncias lícitas e ilícitas, entre 13-15 anos $^{23}$. Estudo realizado na região sudeste do Brasil com usuários internados por consumo de drogas, apontou que $45 \%$ dos homens e $37 \%$ das mulheres iniciaram o uso com menos de 15 anos e maioria abaixo dos 20 anos ${ }^{15}$. Resultados da presente pesquisa corroboram estudos em que foi verificada associação entre consumo precoce e fluxo crescente em idade posterior, caracterizando o fenômeno da escalada das drogas lícitas, para as ilícitas ${ }^{7}$.

Quanto ao padrão de consumo, os achados do presente estudo, que apontam o crack como a droga ilícita mais consumida, ratificam outras pesquisas com usuários adolescentes e adultos jovens, especialmente o sexo masculino ${ }^{15}$. Resultados de diferentes pesquisas constatam que o crack, isolado ou associado à maconha é a droga mais consumida entre usuários de múltiplas SPA, em ambos os sexos, cabendo ressaltar que, em geral, a iniciação ocorre com álcool, tabaco e maconha, cujo consumo sofre aumento significativo em direção aos padrões frequente e pesado/abusivo ${ }^{24}$.

A comparação entre os quatro levantamentos do CEBRID mostrou que o consumo de maconha apresentou crescimento significante, cabendo enfatizar que, embora considerada SPA leve, comparada ao crack e cocaína, a maconha pode acarretar sérias complicações a saúde física e psíquica $^{10-13}$. Os achados da presente pesquisa reiteram esses resultados, considerando as altas taxas de consumo pesado/abusivo da maconha entre adolescentes, comparados aos adultos jovens.

Para finalizar, cabe ressaltar que esta pesquisa se limita ao estudo de uma população, cujos dados corroboram achados de outros estudos, embora não possam ser generalizados. Entretanto, vale salientar que este Centro de Atenção Psicossocial - CAPS AD presta atendimento aos usuários de SPA nos municípios da região do semiárido do Estado da Bahia, com destaque para
Feira de Santana, segunda maior cidade do Estado (600 mil habitantes), portal de migração dos municípios menos desenvolvidos, localizados na região semiárida e sertão baiano.

Os resultados retratados nas características de iniciação e consumo de drogas em um CAPSAD do município de Feira de Santana, aproxima saberes e práticas fundamentadas na Reforma Psiquiátrica Brasileira, com desinstitucionalização de programas e serviços especializados no tratamento aos usuários de SPA, ampliando assim a interface entre a saúde coletiva e a saúde mental. $\mathrm{O}$ estudo pode incentivar novas pesquisas na temática, buscando responder questões que ultrapassam os objetivos do presente estudo, assim como subsidiar ações em serviços que propõem práticas adequadas e eficientes, direcionadas aos usuários de SPA.

\section{Considerações finais}

Os resultados de iniciação e consumo muito precoce das SPA ilícitas entre adolescentes observados neste estudo possibilitam reflexões e sugerem a necessidade de medidas preventivas em fases mais precoces, evitando o avanço do problema em nível mais complexo, cuja morbidade e mortalidade são apresentadas em nível mundial.

O fortalecimento e a ampliação da Política Nacional de Redução de Danos é instrumental importante de garantia de direitos sociais, especialmente o direito à saúde. Para efetivar essa proposta e avaliar a eficácia das estratégias, se faz necessário o contínuo levantamento epidemiológico, cujo sistema de informações e dados viabilizam a elaboração de indicadores que podem subsidiar o planejamento e a elaboração de políticas e ações voltadas ao cuidado dos usuários de SPA, possibilitando que sejam protagonistas das intervenções.

\section{Colaboradores}

MCO Costa, MR Silva, NOA Cruz, MTR Amaral, CC Silva e RC Carvalho participaram igualmente de todas as etapas de elaboração do artigo. 


\section{Referências}

1. Hingson RW, Heeren T, Winter MR. Age at Drinking Onset and Alcohol Dependence. Arch Pediatr Adolesc Med 2006; 160(7):739-746.

2. Andrade TM. Uso de drogas entre adolescentes jovens: perspectivas de prevenção no contexto das relações familiares e da educação à luz dos princípios e práticas de redução de danos. Cien Saude Colet 2007; 12(5):1118-1120.

3. Bègue L, Róche S. Multidimensional social control variables as predictors of drunkenness among french adolescents. J adolesc 2009; 32(2):171-191.

4. Brasil. Ministério da Saúde (MS). Secretaria de Atenção à Saúde. SVS/CN-DST/AIDS. A Política do Ministério da Saúde para Atenção Integral a Usuários de Álcool e outras Drogas. 2a Edição. Rev. Ampl. Brasília: MS; 2004.

5. Silber T, Souza RP. Uso e abuso de drogas na adolescência: o que se deve saber e o que se pode fazer. Adolesc. Latinoam. 1998; 1(3):148-162.

6. Gálduroz JCF. Epidemiologia do uso, abuso e dependência de substâncias psicoativas. In: BRASIL. Secretaria Nacional Antidrogas. Sistema para detecção do uso abusivo e dependência de substâncias psicoativas (SUPERA):encaminhamento, intervenção breve, reinserção social e acompanhamento. Módulo $1-O$ uso de substâncias psicoativas no Brasil: epidemiologia, legislação, políticas públicas e fatores culturais. Brasília: Secretaria Nacional Antidrogas; 2006. p. 13-23.

7. Muza GM, Bettiol H, Muccillo G, Barbieri MA. Consumo de substâncias psicoativas por adolescentes escolares de Ribeirão Preto, SP. I - Prevalência do consumo por sexo, idade e tipo de substância. Rev Saude Publica 1997; 31(1):21-29.

8. Cleveland MJ, Feinberg ME, Bontempo DE, Greenberg MT. The role of risk and protective factors in substance use across adolescence. J Adolesc Health 2008; 43(2):157-164.

9. Silva LVER, Malbergier A, Stempliuk VA, Andrade AG. Fatores associados ao consumo de álcool e drogas entre estudantes universitários. Rev Saude Publica 2006; 40(2):280-288.

10. Carlini EA, Galduróz JCF, Noto AR, Nappo SA. I Levantamento Domiciliar Sobre o Uso de Drogas Psicotrópicas no Brasil - 2001. 1 a Edição. São Paulo: CLR Balieiro Editores Ltda; 2002.

11. Carlini EA, Galduróz JCF, Noto AR, Fonseca AM, Carlini CM, Oliveira LG. II Levantamento Domiciliar sobre o Uso de Drogas Psicotrópicas no Brasil. $1^{\text {a }}$ Edição. São Paulo: Páginas \& Letras Editora e Gráfica Ltda; 2007. p. 1-472.

12. Galduróz JCF, Noto AR, Carlini EA. IV Levantamento sobre o uso de drogas entre estudantes de $1^{\circ} \mathrm{e} 2^{\circ}$ graus em 10 capitais brasileiras. 2a Edição. São Paulo: Centro Brasileiro de Informações sobre Drogas Psicotrópicas (CEBRID), UNIFESP; 1997.

13. Galduróz JCF, Noto AR, Fonseca AM, Carlini EA. $\mathrm{V}$ Levantamento nacional sobre o consumo de drogas psicotrópicas entre estudantes do ensino fundamental e médio da rede pública de ensino nas 27 capitais brasileiras. São Paulo: Editora Balieiro; 2005.
14. Tavares BF, Béria JU, Lima MS. Prevalência do uso de drogas e desempenho escolar entre adolescentes. Rev Saude Publica 2001; 35(2):150-158.

15. Borini P, Guimarães RC, Borini SB. Usuários de drogas ilícitas internados em Hospital Psiquiátrico: padrões de uso e aspectos demográficos e epidemiológicos. J Bras Psiquiatr 2003; 52(3):171-179.

16. Schenker M, Minayo MCS. Fatores de risco e de proteção para o uso de drogas na adolescência. Cien Saude Colet 2005; 10(3):707-717.

17. Costa MCO, Gomes WA. Crescimento e Desenvolvimento na Infância e na Adolescência. In: Costa MCO, Souza RP, editores. Semiologia e Atenção Primária à Criança e ao Adolescente. Porto Alegre: Artmed; 2005. p. 17-39.

18. Sanchez ZM, Nappo SA. Seqüência de drogas consumidas por usuários de crack e fatores interferentes. Rev Saude Publica 2002; 36(4):420-430.

19. Passos SRL, Camacho LAB. Características da clientela de um centro de tratamento para dependência de drogas. Rev Saude Publica 1998; 32(1):64-71.

20. Kuntsche EN, Kuendig H. What is Worse? A Hierarchy of Family-Related Risk Factors Predicting Alcohol Use in Adolescence. Subst. Use Misuse 2006; 41(1):71-86.

21. Marques ACPR, Cruz MS. O adolescente e o uso de drogas. Rev Bras Psiquiatr 2000; 22(Supl. II):32-36.

22. Patrick ME, Collins LM, Smith E, Caldwell L, Flisher A, Wegner L. Prospective Longitudinal Model of Substance Use Onset among South African Adolescents. Subst. Use Misuse. 2009; 44(5):647-662.

23. Ferigolo M, Barbosa FB, Arbo E, Malysx AS, Stein AT, Barros HMT. Prevalência do consumo de drogas na FEBEM, Porto Alegre. Rev Bras Psiquiatr 2004; 26(1):10-16.

24. Soldera M, Dalgalarrondo P, Corrêa Filho HR, Silva CAM. Uso de drogas psicotrópicas por estudantes: prevalência e fatores sociais associados. Rev Saude Publica 2004; 38(2):277-283.

Artigo apresentado em 03/09/2013

Aprovado em 20/10/2013

Versão final apresentada em 29/10/2013 